\title{
Einwirkung von Stosswellen auf tierische Zellkulturen Durchflußzytometrische Untersuchungen physiologischer Parameter
}

\author{
F.Brümmer, J.Brenner, Th.Bräuner, M.Nesper, D.F.Hülser
}

Abteilung Biophysik, Biologisches Institut der Universität Stuttgart

Ziel unserer Arbeiten ist die Charakterisierung biologischer und physikalischer Eigenschaften von Stoßwellen sowie die Abgrenzung primärer Stoßwellenwirkungen von sekundären Wirkungen auf biologisches Material. Für unsere Untersuchungen verwendeten wir Maus-LeukämieZellen (L1210).

Zur Erzeugung von Stoßwellen benutzen wir einen Experimentallithotripter (Typ XL 1) der Firma Dornier Medizintechnik. Dabei wird die in einem Hochspannungskondensator gespeicherte Energie (18 kV, $80 \mathrm{nF})$ uber eine Unterwasserfunkenstrecke (Elektrode), die im Brennpunkt (F1) eines Messing-Halbellipsoids lokalisiert ist, entladen. Uber das Halbellipsoid wird die Stoßwelle im zweiten Brennpunkt (F2) fokussiert, in dem sich die Zellen in einer Polyethylen-Pipette befinden.

An Kunststeinen konnten wir in teilweise entgastem Wasser eine größere Schädigung als in normalem Wasser feststellen, was auch an den Zellsuspensionen zu beobachten war. Durch eine Doppelfluoreszenzfärbung (Fluoresceindiacetat/Propidiumjodid) wurden die Einzelzellsuspensionen auf das Verhältnis lebende/tote Zellen im Durchflußzytometer untersucht. Gleichzeitig wurde der Anteil zerstörter Zellen in einem elektronischen Zellzählgerät (Coulter Counter) ermittelt.

Diese Messungen ergaben eine deutliche Abnahme intakter Zellen mit steigender Stoßwellenzahl und eine $L_{50}$ von 500 Stoßwellen. Die verbleibende Population geometrisch intakter Zellen enthielt jedoch noch eine Subpopulation, die sich mit der Doppelfluoreszenzfärbung als tote Zellen herausstellte.

Die Ergebnisse belegen eine dosis-abhängige Abnahme lebender Zellen bzw. Zunahme toter Zellen.

Nach einer Stoßwellenbehandlung immobilisierter L1210-Zellen war keine Schädigung bzw. DosisAbhängigkeit feststellbar. Dies zeigt deutlich, da $\beta$ andere Mechanismen als die reine Stoßwellenwirkung für den hohen Grad der Zellschädigung an Zellsuspensionen verantwortlich sein müssen. Hierfür kommen neben Beschleunigungs- und
Scherkräften vor allem Kavitationen in Frage. Auf einer dunnen Aluminiumfolie lassen sich im Fokusbereich deutliche Einschläge nach Stoßwellenapplikation in teilweise entgastem Wasser nachweisen, die auf Kavitationen hinweisen. Gemeinsame Versuche zusammen mit Herrn Prof. Eisenmenger und Dipl.-Phys. Staudenraus am Physikalischen Institut der Universität Stuttgart zeigten, da $\beta$ im Gegensatz dazu in Gelatine keine Kavitationen auf traten.

Darüber hinaus zeigten sich deutliche Unterschiede im Grad der Schädigung je nach Temperatur des Lithotripter-Wasserbades und des Gasgehaltes im Wasser. 